\title{
On the ground state wave function of matrix theory
}

\author{
Ying-Hsuan Lin and Xi Yin \\ Jefferson Physical Laboratory, Harvard University, \\ Cambridge, MA 02138 U.S.A. \\ E-mail: yhlin@physics.harvard.edu, xiyin@fas.harvard.edu
}

ABSTRACT: We propose an explicit construction of the leading terms in the asymptotic expansion of the ground state wave function of BFSS SU $(N)$ matrix quantum mechanics. Our proposal is consistent with the expected factorization property in various limits of the Coulomb branch, and involves a different scaling behavior from previous suggestions. We comment on some possible physical implications.

KEYworDS: Supersymmetric gauge theory, Field Theories in Lower Dimensions, M(atrix) Theories, M-Theory

ARXIV EPRINT: 1402.0055 


\section{Contents}

1 Introduction 1

2 The asymptotic expansion 3

2.1 Removing the gauge redundancy 3

2.2 The asymptotic expansion of the supercharge 5

2.3 Reducing to the Cartan wave function 6

2.4 Treating the Cartan fermions 8

3 The leading ground state wave function $\quad 8$

3.1 Reducing to free superparticles 8

3.2 The $\mathrm{SU}(N)$ proposal 9

4 Going to higher orders 12

$\begin{array}{lll}4.1 & \text { The general structure } & 12\end{array}$

$\begin{array}{lll}4.2 & \text { Solving for } \Psi_{1} & 13\end{array}$

$\begin{array}{lll}4.3 & \text { Higher orders in the } r^{-\frac{3}{2}} \text { expansion } & 14\end{array}$

5 Discussion $\quad 14$

$\begin{array}{ll}\text { A Change of variables in the asymptotic expansion } & 16\end{array}$

\section{Introduction}

The matrix theory of Banks-Fischler-Susskind-Shenker [1, 2] was formulated by [4-6] along the lines of the AdS/CFT correspondence [3] as a duality between the 16-supercharge $\mathrm{SU}(N)$ gauged matrix quantum mechanics and the decoupling limit of the 0-brane geometry in type IIA string theory, which admits an M-theory lift to an asymptotically null-compactified spacetime. Though the matrix quantum mechanics may appear to be a (deceivingly) simple theory, it has been difficult to extract bulk physics from it. Perturbative computations in matrix theory beyond one-loop suffers from infrared divergences that are regularized through non-perturbative effects [8]. It is expected that semi-classical gravity in the bulk can only be recovered through strong coupling dynamics at large $N$. Relatively little is known regarding the strong coupling/low energy dynamics of matrix quantum mechanics beyond Monte Carlo simulations. Attempts of analytically understanding the strong coupling dynamics of matrix theory include the use of truncated Schwinger-Dyson equations, with limited success.

Various indirect arguments, as well as a careful computation of the supersymmetric index, indicate that the theory has a unique, normalizable, $\mathrm{SO}(9)$ rotationally invariant 
supersymmetric ground state [11-16]. There is a continuum of scattering states above the ground state. It is commonly believed (though not often stated explicitly) that there are no normalizable energy eigenstates of nonzero energy; in other words, all excited energy eigenstates are scattering states. This is consistent with the bulk picture that black holes can decay by radiating D0-branes [29], which are the only particles in the bulk that can escape to infinity. The bulk picture on the other hand also suggests the existence of an exponentially large number of metastable states with exponentially long life time. ${ }^{1}$ These metastable states are the dual description of the microstates of the black hole at finite temperature.

An outstanding question is to describe these metastable states directly in the framework of matrix quantum mechanics. The first step is to understand the structure of the ground state wave function. An asymptotic expansion for the ground state wave function in the $\mathrm{SU}(2)$ case has been studied in [17, 18], and subsequent proposals for $N \geq 3$ were made in $[19,20]$. In this paper we extend the study of the asymptotic expansion to the general $\mathrm{SU}(N)$ matrix theory. We will demonstrate that, first of all, the leading term in the asymptotic ground state wave function is governed by a set of 16 supercharges that describe $N$ or $N-1$ free non-relativistic superparticles on $\mathbb{R}^{9 \mid 16}$. This is intuitive from the perspective of effective field theory on the Coulomb branch, though in the EFT approach it was unclear how to carry out a systematic expansion in $1 / r$, particularly due to trouble with infrared divergences.

We then propose an explicit form of the leading asymptotic ground state wave function, based on a structure that involves a summation over trees that successively group the $N$ particles. Our proposed form solves the supercharge constraint exactly, and obeys the expected factorization property in various limits on the Coulomb branch of the theory. There is a small ambiguity in our wave function, encoded in a simple set of constant "twobody coefficients", which are not determined by any simple argument we know of. Our proposal differs from previous suggestions in the $\mathrm{SU}(3)$ case [19]; in particular, the overall scaling power with $r$ is different (the proposal of [19] tails off faster at large distances by a factor of $r^{-14}$ ). We also compute the next-to-leading order correction to the asymptotic wave function, and show how we can go to higher orders.

Let us begin by recalling the Hamiltonian of matrix theory,

$$
H=\frac{1}{2} \operatorname{Tr}\left(P_{i}^{2}-\frac{1}{2}\left[X^{i}, X^{j}\right]^{2}-\widehat{\Theta}^{T} \Gamma^{i}\left[X^{i}, \widehat{\Theta}\right]\right),
$$

where the bosonic and fermionic matrices can be written as $X^{i}=X_{A}^{i} T_{A}, \widehat{\Theta}_{\alpha}=\widehat{\Theta}_{\alpha A} T_{A}$, with $T_{A}$ the $\mathrm{SU}(N)$ generators, normalized by $\operatorname{Tr}\left(T_{A} T_{B}\right)=\delta_{A B}$. Here $i=1,2, \cdots, 9$ and $\alpha=1, \cdots, 16$ are vector and spinor indices of $\mathrm{SO}(9) . P_{i}$ are the canonical momenta conjugate to $X^{i}$, while $\widehat{\Theta}_{\alpha A}$ obey canonical anti-commutation relations

$$
\left\{\widehat{\Theta}_{\alpha A}, \widehat{\Theta}_{\beta B}\right\}=\delta_{\alpha \beta} \delta_{A B} .
$$

\footnotetext{
${ }^{1}$ This is a peculiar feature of the bulk geometry, in that only the D0-branes can approach asymptotic infinity at a finite cost of energy. It is in contrast to Schwarzschild black holes in flat spacetime whose lifetime scales like a power of its mass.
} 
Gauging the $\mathrm{SU}(N)$ means that we restrict the Hilbert space to consist of $\mathrm{SU}(N)$ invariant states. The 16 supercharges are written as

$$
Q_{\alpha}=\operatorname{Tr}\left(P_{i}\left(\Gamma^{i} \widehat{\Theta}\right)_{\alpha}-\frac{i}{2}\left[X^{i}, X^{j}\right]\left(\Gamma^{i j} \widehat{\Theta}\right)_{\alpha}\right),
$$

which obey the supersymmetry algebra up to a gauge rotation

$$
\left\{Q_{\alpha}, Q_{\beta}\right\}=2 \delta_{\alpha \beta} H+2 \Gamma_{\alpha \beta}^{i} X_{A}^{i} C_{A} .
$$

Here $C_{A}$ are the operator realization of $\mathrm{SU}(N)$ generators,

$$
C=C_{A} T_{A}=-i\left[X^{i}, P_{i}\right]-\frac{1}{2}\left\{\widehat{\Theta}_{\alpha}, \widehat{\Theta}_{\alpha}\right\}
$$

Our objective is to find the $\mathrm{SO}(9)$ invariant ground state wave function annihilated by all $Q_{\alpha}$. The idea is to begin with a Born-Oppenheimer-type approximation, by starting at a generic point on the Coulomb branch where the $X^{i}$ 's are close to being commuting with one another, and treat the off-diagonal components as internal degrees of freedom. In the next section we will formulate an expansion of the wave function in powers of $r^{-\frac{3}{2}}$ where $r$ is essentially the distance between eigenvalues on the Coulomb branch. A (so far) consistent proposal for the leading term in the asymptotic expansion of the ground state is given in section 3. The next-to-leading order correction is computed in section 4 , and a systematic way of going to higher orders is presented. We conclude with discussions on the physical implications of our result and some speculations.

\section{The asymptotic expansion}

In this section we explain the method for solving the supersymmetry constraint equations on the wave function based on an asymptotic expansion, closely following the approach of [18] (see also [20]).

\subsection{Removing the gauge redundancy}

We are after the $\mathrm{SU}(N)$-invariant ground state wave function which is annihilated by the supercharges $Q_{\alpha}$, namely

$$
\operatorname{Tr}\left\{\frac{\partial}{\partial X^{i}} \Gamma_{\alpha \beta}^{i} \widehat{\Theta}_{\beta}+\frac{1}{2}\left[X^{i}, X^{j}\right] \Gamma_{\alpha \beta}^{i j} \widehat{\Theta}_{\beta}\right\} \Psi=0 .
$$

In analyzing the asymptotic form of the wave function, we will expand near a generic point at large distances on the Coulomb branch, and put the bosonic matrices $X^{i}$ in the form

$$
U X^{i} U^{-1}=\left(\begin{array}{cccc}
r_{1}^{i} & 0 & & \\
0 & r_{2}^{i} & & \\
& & \ddots & \\
& & & r_{N}^{i}
\end{array}\right)+\left(\begin{array}{cccc}
0 & q_{12}^{i} & & \\
\left(q_{12}^{i}\right)^{*} & 0 & & \\
& & \ddots & \\
& & & 0
\end{array}\right)
$$


for some $\mathrm{SU}(N)$ matrix $U$. We write $\vec{r}_{a}=\left(r_{a}^{1}, \cdots, r_{a}^{9}\right), \vec{q}_{a b}=\left(q_{a b}^{1}, \cdots, q_{a b}^{9}\right)$, and work in the regime of large $\left|\vec{r}_{a}-\vec{r}_{b}\right|$ such that $q_{a b}^{i}$ are very massive. To ensure that this is the case, namely that the $q_{a b}^{i}$ 's are transverse to the valley of the scalar potential, we must choose $U$ in such a way that $\vec{q}_{a b} \cdot\left(\vec{r}_{a}-\vec{r}_{b}\right)=0$ for all $a, b$. This condition fixes $U$ up to the diagonal $\mathrm{U}(1)^{N-1}$ that rotates the phases of $\vec{q}_{a b}$. We will leave these degrees of freedom in $U$ unfixed. This is acceptable because it still allows us to work in the regime of small $q_{a b}^{i}$ in the large $r_{a}^{i}$ limit. Since in this limit $q_{a b}^{i}$ are described as harmonic oscillators in a potential $\left|\vec{r}_{a}-\vec{r}_{b}\right|^{2}\left(q_{a b}^{i}\right)^{2}$, it is convenient to define

$$
y_{a b}^{i}=\left|\vec{r}_{a}-\vec{r}_{b}\right|^{\frac{1}{2}} q_{a b}^{i}
$$

so that $y_{a b}^{i} \sim \mathcal{O}(1)$.

Similarly, we separate $\widehat{\Theta}_{\alpha}$, after the appropriate $\mathrm{SU}(N)$ rotation, into diagonal and off-diagonal modes, according to

$$
U \widehat{\Theta}_{\alpha} U^{-1}=\left(\begin{array}{cccc}
\theta_{\alpha 1} & 0 & & \\
0 & \theta_{\alpha 2} & & \\
& & \ddots & \\
& & & \theta_{\alpha N}
\end{array}\right)+\left(\begin{array}{cccc}
0 & \left(\Theta_{\alpha}\right)_{12} & \\
\left(\Theta_{\alpha}\right)_{12}^{*} & 0 & & \\
& & \ddots & \\
& & & 0
\end{array}\right)
$$

From now the unhatted notation $\left(\Theta_{\alpha}\right)_{a b}$ will always refer to these off-diagonal components of $U \widehat{\Theta}_{\alpha} U^{-1}$. Note that the overall $\mathrm{SU}(N)$ gauge rotation, which acts on both $X^{i}$ and $\widehat{\Theta}_{\alpha}$, only acts by rotating $U$ and does not act on $\left(r^{i}, q^{i}, \theta_{\alpha}, \Theta_{\alpha}\right)$.

The next step is to write $\partial / \partial X^{i}$ in terms of derivatives on $r_{a}^{i}$ and $y_{a b}^{i}$. The details are given in appendix $\mathrm{A}$, with the result

$$
\begin{aligned}
{\left[U \frac{\partial}{\partial X^{i}} U^{-1}\right]_{b a}=} & \delta_{a b} \frac{\partial}{\partial r_{a}^{i}}+\Pi_{a b}^{i j} \frac{\partial}{\partial q_{a b}^{j}}-\frac{\widehat{r}_{a b}^{i}}{\left|r_{a b}\right|} \sum_{c \neq a, b}\left(\frac{y_{c a}^{k}}{\left|r_{c a}\right|^{\frac{1}{2}}} \Pi_{c b}^{k j} \frac{\partial}{\partial q_{c b}^{j}}-\frac{y_{b c}^{k}}{\left|r_{b c}\right|^{\frac{1}{2}}} \Pi_{a c}^{k j} \frac{\partial}{\partial q_{a c}^{j}}\right) \\
& +\frac{\widehat{r}_{a b}^{i}}{\left|\vec{r}_{a b}\right|}\left[U \frac{\partial}{\partial U}\right]_{b a}+\mathcal{O}\left(r^{-\frac{5}{2}}\right),
\end{aligned}
$$

where $r_{a b}^{i} \equiv r_{a}^{i}-r_{b}^{i}$, and $\Pi_{a b}^{i j} \equiv \delta^{i j}-\widehat{r}_{a b}^{i} \widehat{r}_{a b}^{j}$. Next, we need to change coordinate on the fermions $\widehat{\Theta}_{\alpha}$ into $\left(\theta_{\alpha}, \Theta_{\alpha}\right)$ as well. In doing so, we must make the replacement

$$
\left[U \frac{\partial}{\partial U}\right]_{a b} \rightarrow R_{a b}+M_{a b}
$$

where $R_{a b}$ is the overall $\mathrm{SU}(N)$ gauge rotation generator that only acts on $U$ but not on $\left(r^{i}, q^{i}, \theta_{\alpha}, \Theta_{\alpha}\right)$, and $M_{a b}$ is the $\mathrm{SU}(N)$ generator acting on the fermions. ${ }^{2}$

Now we can write

$$
\begin{aligned}
{\left[U \frac{\partial}{\partial X^{i}} U^{-1}\right]_{b a}=} & \delta_{a b} \frac{\partial}{\partial r_{a}^{i}}+\Pi_{a b}^{i j} \frac{\partial}{\partial q_{a b}^{j}}-\frac{\widehat{r}_{a b}^{i}}{\left|r_{a b}\right|} \sum_{c \neq a, b}\left(\frac{y_{c a}^{k}}{\left|r_{c a}\right|^{\frac{1}{2}}} \Pi_{c b}^{k j} \frac{\partial}{\partial q_{c b}^{j}}-\frac{y_{b c}^{k}}{\left|r_{b c}\right|^{\frac{1}{2}}} \Pi_{a c}^{k j} \frac{\partial}{\partial q_{a c}^{j}}\right) \\
& +\frac{\widehat{r}_{a b}^{i}}{\left|r_{a b}\right|}\left(R_{b a}+M_{b a}\right)+\mathcal{O}\left(r^{-\frac{5}{2}}\right) .
\end{aligned}
$$

\footnotetext{
${ }^{2}$ Explicitly,

$$
M_{a b}=\frac{1}{2}\left[\left(\Theta_{\alpha}\right)_{a e},\left(\Theta_{\alpha}\right)_{e c}\right]+\left(\theta_{\alpha a}-\theta_{\alpha b}\right)\left(\Theta_{\beta}\right)_{a b} .
$$
}


In the application below, we will take this expression for $\partial / \partial X^{i}$ to act on an $\mathrm{SU}(N)$ invariant wave function, that is, a wave function that is invariant under the $\mathrm{SU}(N)$ action simultaneously on the original bosons and fermions $X^{i}$ and $\Theta_{\alpha}$. In the new coordinate system $\left(U, r^{i}, q^{i}, \theta_{\alpha}, \Theta_{\alpha}\right)$, it only acts on $U$. The upshot is that $R_{a b}$ annihilates the $\operatorname{SU}(N)$ invariant wave function and can be dropped from now, and $U$ will no longer appear explicitly in our computations below.

\subsection{The asymptotic expansion of the supercharge}

After dropping the $R_{a b}$ term and changing variables from $q_{a b}^{i}$ to $y_{a b}^{i}$, we can now write the supercharge as an expansion in $r^{-\frac{3}{2}}$, in the form

$$
\begin{aligned}
i Q_{\alpha}= & \sum_{a \neq b}\left|\vec{r}_{a b}\right|^{\frac{1}{2}}\left[\Pi_{a b}^{i j} \frac{\partial}{\partial y_{b a}^{j}} \Gamma_{\alpha \beta}^{i}\left(\Theta_{\beta}\right)_{b a}+\frac{1}{2} \widehat{r}_{a b}^{i} y_{a b}^{j} \Gamma_{\alpha \beta}^{i j}\left(\Theta_{\beta}\right)_{b a}\right] \\
& +\sum_{a} \frac{\partial}{\partial r_{a}^{i}} \Gamma_{\alpha \beta}^{i} \theta_{\beta a}+\sum_{a \neq b}\left[\frac{\widehat{r}_{a b}^{i}}{2\left|r_{a b}\right|} y_{a b}^{j} \frac{\partial}{\partial y_{a b}^{j}} \Gamma_{\alpha \beta}^{i}\left(\theta_{\beta a}-\theta_{\beta b}\right)\right. \\
& +\sum_{c \neq a, b} \frac{y_{a c}^{i} y_{c b}^{j}}{\left|r_{a c}\right|^{\frac{1}{2}}\left|r_{b c}\right|^{\frac{1}{2}}} \Gamma_{\alpha \beta}^{i j}\left(\Theta_{\beta}\right)_{b a}+\frac{y_{a b}^{i} y_{b a}^{j}}{2\left|r_{a b}\right|} \Gamma_{\alpha \beta}^{i j}\left(\theta_{\beta a}-\theta_{\beta b}\right)-\frac{\widehat{r}_{a b}^{i}}{\left|r_{a b}\right|} \Gamma_{\alpha \beta}^{i}\left(\Theta_{\beta}\right)_{b a} M_{a b} \\
& \left.-\sum_{c \neq a, b}\left(\frac{\left|r_{b c}\right|^{\frac{1}{2}}}{\left|r_{a c}\right|^{\frac{1}{2}}} y_{c a}^{k} \Pi_{c b}^{k j} \frac{\partial}{\partial y_{c b}^{j}}-\frac{\left|r_{a c}\right|^{\frac{1}{2}}}{\left|r_{b c}\right|^{\frac{1}{2}}} y_{b c}^{k} \Pi_{a c}^{k j} \frac{\partial}{\partial y_{a c}^{j}}\right) \frac{\widehat{r}_{a b}^{i}}{\left|r_{a b}\right|} \Gamma_{\alpha \beta}^{i}\left(\Theta_{\beta}\right)_{a b}\right]+\mathcal{O}\left(r^{-\frac{5}{2}}\right) .
\end{aligned}
$$

We will write the first line after the equal sign as $Q_{\alpha}^{0}$ and $^{3}$ the next two lines as $Q_{\alpha}^{1}$. $Q_{\alpha}^{0}$ scales like $r^{\frac{1}{2}}$ while $Q_{\alpha}^{1}$ scales like $r^{-1}$. The wave function will take the following form

$$
\Psi=\Psi_{0}+\Psi_{1}+\Psi_{2}+\cdots,
$$

where $\Psi_{n}$ scales like $r^{-\kappa-\frac{3}{2} n}$. Our goal is to determine $\kappa$. Separating the equations according to the scaling degree in $r$, we have a series of equations

$$
\begin{aligned}
& Q_{\alpha}^{0} \Psi_{0}=0, \\
& Q_{\alpha}^{0} \Psi_{1}+Q_{\alpha}^{1} \Psi_{0}=0, \quad \text { etc. }
\end{aligned}
$$

The first equation is a differential equation in $y_{a b}^{i}$ only. The solution $\Psi_{0}$ takes the form

$$
\Psi_{0}=f\left(\vec{r}_{a}\right)\left|\psi_{0}(\widehat{r})\right\rangle_{y, \Theta},
$$

where $\left|\psi_{0}(\widehat{r})\right\rangle_{y, \Theta}$ is the ground state wave function of an $\widehat{r}_{a b}$-dependent (denoted here collectively by $\widehat{r})$ supersymmetric harmonic oscillator in the off-diagonal $(y, \Theta)$ sector, obeying

$$
Q_{\alpha}^{0}\left|\psi_{0}(\hat{r})\right\rangle_{y, \Theta} \equiv \sum_{a \neq b}\left|r_{a b}\right|^{\frac{1}{2}}\left[\Pi_{a b}^{i j} \frac{\partial}{\partial y_{b a}^{j}} \Gamma_{\alpha \beta}^{i}\left(\Theta_{\beta}\right)_{b a}+\frac{1}{2} \widehat{r}_{a b}^{i} y_{a b}^{j} \Gamma_{\alpha \beta}^{i j}\left(\Theta_{\beta}\right)_{b a}\right]\left|\psi_{0}(\hat{r})\right\rangle_{y, \Theta}=0
$$

$f\left(\vec{r}_{a}\right)$ is a so far undetermined wave function that has some overall scaling $r^{-\kappa}$, and includes the fermionic wave function in the diagonal $\theta$ sector.

\footnotetext{
${ }^{3}$ Our convention for $Q_{\alpha}^{n}$ 's differs from that of the usual supercharge by a factor of $i$.
} 
The next key step is to consider a projection $P_{0}$ onto the zero-eigenspace of $Q_{\alpha}^{0}$. Since $i Q_{\alpha}^{0}$ is Hermitian, any state of the form $Q_{\alpha}^{0} \Psi_{1}$ must be orthogonal to the zero-eigenspace of $Q_{\alpha}^{0}$, and is thus annihilated by $P_{0}$. Consequently, the next-to-leading order equation in the asymptotic expansion implies

$$
P_{0} Q_{\alpha}^{1} \Psi_{0}=0
$$

Since $Q_{\alpha}^{1}$ involves an $r$-derivative, this equation will provide nontrivial constraints on $f\left(\vec{r}_{a}\right)$.

\subsection{Reducing to the Cartan wave function}

$Q_{\alpha}^{0}$ has an anti-commutator of the form

$$
\left\{Q_{\alpha}^{0}, Q_{\beta}^{0}\right\}=\delta_{\alpha \beta} \sum_{a \neq b}\left|r_{a b}\right|\left[\Pi_{a b}^{i j} \frac{\partial}{\partial y_{a b}^{i}} \frac{\partial}{\partial y_{b a}^{j}}-\frac{1}{4} y_{a b} \cdot y_{b a}-\frac{1}{2} \widehat{r}_{a b}^{k} \Gamma_{\gamma \delta}^{k}\left(\Theta_{\gamma}\right)_{a b}\left(\Theta_{\delta}\right)_{b a}\right]+\Gamma_{\alpha \beta}^{k} \mathcal{M}_{k} .
$$

For each pair $a, b$, consider the matrix $\widehat{r}_{a b}^{k} \Gamma_{\alpha \beta}^{k}$ that acts on $\mathrm{SO}(9)$ spinors. This matrix has eight +1 eigenvalues and eight -1 eigenvalues. Let $\Pi_{a b}^{ \pm}$be the projection operators onto the positive and negative spinor eigenspaces of $\widehat{r}_{a b}^{i} \Gamma^{i}$. By definition, $\Pi_{a b}^{ \pm}=\Pi_{b a}^{\mp}$.

Given a fixed pair $a, b$, let $\left|F_{a b}\left(\widehat{r}_{a b}\right)\right\rangle$ be a unit norm state in the $\Theta_{a b}$ sector, that is annihilated by $\left(\Theta_{a b}^{-}\right)_{\alpha} \equiv\left(\Pi_{a b}^{-}\right)_{\alpha \beta}\left(\Theta_{\beta}\right)_{a b}$ and $\left(\Theta_{b a}^{-}\right)_{\alpha} \equiv\left(\Pi_{b a}^{-}\right)_{\alpha \beta}\left(\Theta_{\beta}\right)_{b a}=\left(\Pi_{a b}^{+}\right)_{\alpha \beta}\left(\Theta_{\beta}\right)_{a b}^{*}$ for all $\alpha$, and is invariant under simultaneous $\mathrm{SO}(9)$ rotations on $\widehat{r}_{a b}, \Theta_{a b}$ and $\Theta_{b a}$. Such a state is unique up an overall ( $\widehat{r}$-independent) phase. We will write $|F(\widehat{r})\rangle=\bigotimes_{a<b}\left|F_{a b}\left(\widehat{r}_{a b}\right)\right\rangle$ for such a zeroth-order fermion ground state in the entire off-diagonal $\Theta$ sector (again, the notation here is such that $\widehat{r}$ stands collectively for the set of all $\widehat{r}_{a b}$ 's). We can then construct $\left|\psi_{0}(\widehat{r})\right\rangle$ by combining $|F(\widehat{r})\rangle$ with the harmonic oscillator ground state wave function for the $y_{a b}^{i}$ 's,

$$
\left|\psi_{0}(\widehat{r})\right\rangle=e^{-\frac{1}{4} \sum_{a \neq b}\left|y_{a b}\right|^{2}}|F(\widehat{r})\rangle .
$$

There are $8 N(N-1)$ independent $y_{a b}^{i}$ 's, and the ground state energy of the harmonic oscillator precisely cancels with the fermionic contribution in the coefficient of $\delta_{\alpha \beta}$. One can verify that $\left|\psi_{0}(\widehat{r})\right\rangle$ is annihilated by $\mathcal{M}_{k}$ as well.

Now we can write

$$
\Psi_{0}=e^{-\frac{1}{4} \sum_{a \neq b}\left|y_{a b}\right|^{2}} \sum_{s} f_{s}\left(\vec{r}_{a}\right)|s\rangle \otimes|F(\widehat{r})\rangle
$$

for a set of functions $f_{s}\left(\vec{r}_{a}\right)$, where $s$ labels states in the Clifford module of the 16(N-1) diagonal $\theta_{\alpha a}$ 's $\left(s=1, \cdots, 2^{8(N-1)}\right)$. Let us inspect the action of

$$
\begin{aligned}
Q_{\alpha}^{1}= & \sum_{a} \frac{\partial}{\partial r_{a}^{i}} \Gamma_{\alpha \beta}^{i} \theta_{\beta a}+\sum_{a \neq b}\left[\frac{\widehat{r}_{a b}^{i}}{2\left|r_{a b}\right|} y_{a b}^{j} \frac{\partial}{\partial y_{a b}^{j}} \Gamma_{\alpha \beta}^{i}\left(\theta_{\beta a}-\theta_{\beta b}\right)+\sum_{c \neq a, b} \frac{y_{a c}^{i} y_{c b}^{j}}{\left|r_{a c}\right|^{\frac{1}{2}}\left|r_{b c}\right|^{\frac{1}{2}}} \Gamma_{\alpha \beta}^{i j}\left(\Theta_{\beta}\right)_{b a}\right. \\
& +\frac{y_{a b}^{i} y_{b a}^{j}}{2\left|r_{a b}\right|} \Gamma_{\alpha \beta}^{i j}\left(\theta_{\beta a}-\theta_{\beta b}\right)-\frac{\widehat{r}_{a b}^{i}}{\left|r_{a b}\right|} \Gamma_{\alpha \beta}^{i}\left(\Theta_{\beta}\right)_{b a} M_{a b} \\
& \left.-\sum_{c \neq a, b}\left(\frac{\left|r_{b c}\right|^{\frac{1}{2}}}{\left|r_{a c}\right|^{\frac{1}{2}}} y_{c a}^{k} \Pi_{c b}^{k j} \frac{\partial}{\partial y_{c b}^{j}}-\frac{\left|r_{a c}\right|^{\frac{1}{2}}}{\left|r_{b c}\right|^{\frac{1}{2}}} y_{b c}^{k} \Pi_{a c}^{k j} \frac{\partial}{\partial y_{a c}^{j}}\right) \frac{\widehat{r}_{a b}^{i}}{\left|r_{a b}\right|} \Gamma_{\alpha \beta}^{i}\left(\Theta_{\beta}\right)_{a b}\right]
\end{aligned}
$$


on $\Psi_{0}$. Keep in mind that $\partial / \partial r_{a}^{i}$ which appears in $Q_{\alpha}^{1}$ acts not only on the functions $f_{s}\left(\vec{r}_{a}\right)$ but on $|F(\widehat{r})\rangle$ as well.

Under the projection $P_{0}$, we can replace $y_{a b} \cdot \partial_{y_{a b}}$ and $y_{a c}^{i} y_{c b}^{j}$ in $Q_{\alpha}^{1}$ by their expectation values in the harmonic oscillator ground state wave function $e^{-\frac{1}{4} \sum_{a, b}\left|y_{a b}\right|^{2}}=e^{-\frac{1}{2} \sum_{a<b}\left|y_{a b}\right|^{2}}$. Furthermore, any term that involves the product of an odd number of $\Theta$ 's when acting on $\Psi_{0}$ cannot preserve the fermion ground state in the $\Theta$ sector, and the result will be annihilated by $P_{0}$. Note that the projector $P_{0}$ does not touch the $\theta_{\alpha a}$ degrees of freedom. Let us define $\left(\Theta_{\alpha}^{ \pm}\right)_{a b} \equiv \Pi_{a b}^{+}\left(\Theta_{\alpha}\right)_{a b}$. All states that survive the $P_{0}$ projection are annihilated by $\Theta_{\alpha}^{-}$, while any state obtained by acting with $\Theta_{\alpha}^{+}$is killed by $P_{0}$. Using the relation

$$
P_{0}\left(\Theta_{\beta}\right)_{b a} M_{a b} \Psi_{0}=P_{0}\left[\left(\Theta_{\beta}\right)_{b a}^{-}, M_{a b}\right] \Psi_{0}=-\left(\Pi_{a b}^{+}\left(\theta_{a}-\theta_{b}\right)\right)_{\beta} \Psi_{0}
$$

we can replace $Q_{\alpha}^{1}$ by a simplified operator

$$
\begin{aligned}
\widetilde{Q}_{\alpha}^{1} & =\sum_{a} \frac{\partial}{\partial r_{a}^{i}} \Gamma_{\alpha \beta}^{i} \theta_{\beta a}-\sum_{a \neq b} \frac{2}{\left|r_{a b}\right|} \widehat{r}_{a b}^{i} \Gamma_{\alpha \beta}^{i}\left(\theta_{\beta a}-\theta_{\beta b}\right)+\sum_{a \neq b} \frac{1}{\left|r_{a b}\right|}\left(\Pi_{a b}^{+}\right)_{\alpha \beta}\left(\theta_{\beta a}-\theta_{\beta b}\right) \\
& =\sum_{a} \frac{\partial}{\partial r_{a}^{i}} \Gamma_{\alpha \beta}^{i} \theta_{\beta a}-\sum_{a \neq b} \frac{3}{\left|r_{a b}\right|} \widehat{r}_{a b}^{i} \Gamma_{\alpha \beta}^{i} \theta_{\beta a}
\end{aligned}
$$

in the sense that

$$
P_{0} Q_{\alpha}^{1} \Psi_{0}=P_{0} \widetilde{Q}_{\alpha}^{1} \Psi_{0}
$$

Furthemore, the $r_{a}^{i}$ dependence of $\Psi_{0}$ may be expressed as dependence on $\left|r_{a b}\right|$ and $\widehat{r}_{a b}$. Under a variation $\delta r_{a}^{i}$, we have

$$
\begin{aligned}
\delta\left|r_{a b}\right| & =\widehat{r}_{a b} \cdot\left(\delta \vec{r}_{a}-\delta \vec{r}_{b}\right), \\
\delta \widehat{r}_{a b} & =\frac{\delta \vec{r}_{a b}-\widehat{r}_{a b}\left(\widehat{r}_{a b} \cdot \delta \vec{r}_{a b}\right)}{\left|r_{a b}\right|} .
\end{aligned}
$$

Thus we can write

$$
\frac{\partial}{\partial r_{a}^{i}}=\sum_{b \neq a}\left(\widehat{r}_{a b}^{i} \frac{\partial}{\partial\left|r_{a b}\right|}+\frac{\widehat{r}_{a b}^{j}}{\left|r_{a b}\right|} R_{a b}^{j i}\right),
$$

where $R_{a b}^{i j}$ is the generator of $\mathrm{SO}(9)$ rotation on $\widehat{r}_{a b}$ for each pair $a, b$. Note that it does not act on the fermions, by definition. In the $\Theta$ sector, the zeroth order ground state wave function $|F(\widehat{r})\rangle$ by construction is invariant under the $\mathrm{SO}(9)$ rotation on $\widehat{r}_{a b}, \Theta_{a b}$, and $\Theta_{b a}$. Let us denote by $F_{a b}^{i j}$ the $\mathrm{SO}(9)$ rotation generator on $\Theta_{a b}$ and $\Theta_{b a}$, namely

$$
F_{a b}^{i j}=\frac{1}{4}\left(\Theta_{a b} \Gamma^{i j} \Theta_{b a}\right) .
$$

Thus when acting on $|F(\widehat{r})\rangle$ with $R_{a b}^{i j}$, we can replace $R_{a b}^{i j}$ by $-F_{a b}^{i j}$. Note that $F_{a b}^{i j}|F(\widehat{r})\rangle=$ $\frac{1}{4}\left(\Theta_{a b}^{+} \Gamma^{i j} \Theta_{b a}^{+}\right)|F(\widehat{r})\rangle$, and is thus annihilated by the projector $P_{0}$. In other words, we can ignore the $\widehat{r}_{a b}$-dependence of $|F(\widehat{r})\rangle$ in computing $P_{0} \widetilde{Q}_{\alpha}^{1} \Psi_{0}$. For this purpose, we might as 
well replace $\widetilde{Q}_{\alpha}^{1}$ by an operator ${ }^{4}$ of the same form as (2.20), but now acting entirely on the "Cartan wave function"

$$
\Psi_{0}^{C}=\sum_{s} f_{s}\left(\vec{r}_{a}\right)|s\rangle
$$

that is just in the $(r, \theta)$ sector. Now the projector $P_{0}$ is no longer needed; the equation $P_{0} Q_{\alpha}^{1} \Psi_{0}=0$ simply reduces to

$$
\widetilde{Q}_{\alpha}^{1} \Psi_{0}^{C}=0
$$

\subsection{Treating the Cartan fermions}

In the simplest $\mathrm{SU}(2)$ case, the indices $a, b$ take values 1 and 2 (and $\vec{r}_{2}=-\vec{r}_{1}$ ). There are $16 \theta_{\alpha}$ 's, giving rise to $2^{8}=256$ states in the $\theta$ sector. With respect to the $\mathrm{SO}(9)$ rotation on the $\theta_{\alpha}$ 's, these 256 states branch into

$$
44 \oplus 84 \oplus \mathbf{1 2 8} .
$$

Here the $\mathbf{4 4}$ is the traceless symmetric tensor representation of $\mathrm{SO}(9)$. The other two irreducible representations of $\mathrm{SO}(9)$ cannot form a singlet by tensoring with a power of the vector representation (coming from $\widehat{r}$ ). The fermion part of the $\mathrm{SO}(9)$ invariant ground state wave function, $|s\rangle$, must thus be constructed from the 44 . Such a state is unique up to the overall factor, namely, it is $|\widehat{r} \widehat{r}\rangle \equiv \widehat{r}^{i} \widehat{r}^{j}\left|s_{i j}\right\rangle$, where $\left|s_{i j}\right\rangle$ is a basis for the 44 . The $\mathrm{SO}(9)$ invariance of the wave function allows us to replace $R^{i j}$ by $-\frac{1}{4}\left(\theta \Gamma^{i j} \theta\right)$ that rotates $\theta$ instead of $\vec{r}$. One can show that

$$
\widehat{r}^{j}\left(\Gamma^{i} \theta\right)_{\alpha}\left(\theta \Gamma^{i j} \theta\right)|\widehat{r} \widehat{r}\rangle=36 \widehat{r}^{i}\left(\Gamma^{i} \theta\right)_{\alpha}|\widehat{r} \widehat{r}\rangle
$$

One then finds that $\widetilde{Q}_{\alpha}^{1} \Psi_{0}^{C}=0$ is solved by $\Psi_{0}^{C}=r^{-6}|\widehat{r} \widehat{r}\rangle$.

The case of general $\mathrm{SU}(N)$ gauge group will be treated in the next section. Note that the integration measure for our wave function $\Psi$ at large $r$ takes the form ${ }^{5}$

$$
\int \prod_{a=1}^{N-1} d^{9} \vec{r}_{a}\left(\prod_{a<b} r_{a b}^{2}\right) r^{-4 N(N-1)} \int \prod_{a \neq b} d^{9} \vec{y}_{a b} \delta\left(\vec{y}_{a b} \cdot \widehat{r}_{a b}\right) .
$$

If the leading asymptotic wave function $\Psi_{0}$ has an overall scaling $r^{-\kappa}$, normalizability then demands $\kappa>-\frac{3}{2}(N-3)(N-1)$.

\section{The leading ground state wave function}

\subsection{Reducing to free superparticles}

We are seeking an $S_{N} \times \mathrm{SO}(9)$ invariant Cartan wave function $\Psi_{0}^{C}$ that is annihilated by

$$
\widetilde{Q}_{\alpha}^{1}=\sum_{a}\left(\frac{\partial}{\partial r_{a}^{i}}-\sum_{b \neq a} \frac{3}{r_{a b}^{2}} r_{a b}^{i}\right) \Gamma_{\alpha \beta}^{i} \theta_{\beta a}
$$

\footnotetext{
${ }^{4} \mathrm{By}$ a slight abuse of notation we will still denote this operator by $\widetilde{Q}_{\alpha}^{1}$.

${ }^{5}$ Here $r_{a b}^{2}$ come from the gauge-fixing, and $r^{-4 N(N-1)}$ comes from the change of variables from $q$ to $y$.
} 
It is convenient to define

$$
\Psi^{\text {new }} \equiv \prod_{a<b}\left|r_{a b}\right|^{-3} \Psi_{0}^{C}
$$

Then the equation for $\Psi^{\text {new }}$ becomes simply $Q_{\alpha}^{\text {new }} \Psi^{\text {new }}=0$, where $Q_{\alpha}^{\text {new }}$ take the form of the supercharges for a set of free superparticles,

$$
Q_{\alpha}^{\text {new }}=\sum_{a} \frac{\partial}{\partial r_{a}^{i}} \Gamma_{\alpha \beta}^{i} \theta_{\beta a} .
$$

We immediately learn that $\Psi^{\text {new }}$ takes the form

$$
\Psi^{\text {new }}=\sum_{s} F_{s}\left(r_{a}^{i}\right)|s\rangle
$$

where $F_{s}\left(r_{a}^{i}\right)$ for each internal fermion state $|s\rangle$ is a harmonic function on $\mathbb{R}^{9(N-1)}$. Indeed, in the $\mathrm{SU}(2)$ case, $\Psi^{\text {new }}=r^{-9} \widehat{r}^{i} \widehat{r}^{j}\left|s_{i j}\right\rangle \propto \partial_{i} \partial_{j} r^{-7}\left|s_{i j}\right\rangle$ is of such form.

\subsection{The $\mathrm{SU}(N)$ proposal}

So far we have been writing the supercharges and the Hamiltonian as if we were dealing with the $\mathrm{U}(N)$ theory. In dealing with the $\mathrm{SU}(N)$ matrix theory, we need to factor out the center of mass degrees of freedom. This is straightforward in the bosonic sector: the wave function when viewed as a function of $\vec{x}_{1}, \cdots, \vec{x}_{N}$ is taken to be invariant under the overall translation $\vec{P}=\sum_{a=1}^{N} \vec{p}_{a}$. Care must be taken in the fermion sector, however, since we have quantized the $\theta_{\alpha a}$ independently, with

$$
\left\{\theta_{\alpha a}, \theta_{\beta b}\right\}=\delta_{a b} \delta_{\alpha \beta} .
$$

We should factor out $\bar{\theta}=\left(\theta_{1}+\theta_{2}+\cdots+\theta_{N}\right) / N$, and only work with the combinations of $\theta$ 's (for instance, $\theta_{a}-\bar{\theta}$ ) that anti-commute with $\bar{\theta}$. In the expression for the supercharge $Q_{\alpha}$ in terms of $r_{a}^{i}, q_{a b}^{i}, \theta_{\alpha a},\left(\Theta_{\alpha}\right)_{a b}$, the only term that involves the center of mass position and fermionic coordinate $\bar{\theta}$ is $\sum_{a=1}^{N} p_{a}^{i} \Gamma^{i} \theta_{a}$, where $p_{a}^{i}=-i \partial / \partial r_{a}^{i}$. In passing to the $\operatorname{SU}(N)$ system, we can separate

$$
\sum_{a=1}^{N} p_{a}^{i} \Gamma^{i} \theta_{a}=P^{i} \Gamma^{i} \bar{\theta}+\sum_{a=1}^{N}\left(p_{a}^{i}-\frac{1}{N} P^{i}\right) \Gamma^{i}\left(\theta_{a}-\bar{\theta}\right),
$$

and simply drop the first term $P^{i} \Gamma^{i} \bar{\theta}$, since $P^{i}$ and $\bar{\theta}$ commute with the remaining terms of the supercharge. The ground state wave function will depend on the relative bosonic coordinates $\vec{x}_{a}-\vec{x}_{b}$, and its fermionic component may be constructed as an element of the Clifford module coming from $\theta_{a}-\bar{\theta}$. Be aware that $\theta_{a}-\bar{\theta}$ do not anti-commute with $\theta_{b}-\bar{\theta}$ for $a \neq b$. Rather, we have

$$
\left\{\theta_{a}-\bar{\theta}, \theta_{b}-\bar{\theta}\right\}=\delta_{a b}-\frac{1}{N}
$$

One can in principle go to a basis in which the anti-commutators become diagonal, and quantize the theory using that basis. However, such a basis is rather inconvenient to work with. Below we will employ a different approach. 
Though the problem of finding $\Psi_{0}$ is reduced to the free problem of finding $\Psi^{C}$ or $\Psi^{\text {new }}$, this problem doesn't have a unique solution in the general $\mathrm{SU}(N)$ case, even after imposing $S_{N} \times \mathrm{SO}(9)$ invariance. It is possible that there are more constraints coming from the smoothness of the wave function at small $r_{a b}^{i}$ when all order corrections are included. For now, we will constrain $\Psi_{0}$ further by some physical intuition. Namely, we expect that in a limit on the Coulomb branch where $\left(r_{a}^{i}, \theta_{\alpha}\right)$ are separated into two clusters centered at $\left(x^{i}, \theta_{\alpha}\right)$ and $\left(y^{i}, \eta_{\alpha}\right)$, and the $\mathrm{SU}(N)$ broken into $\mathrm{SU}(M) \times \mathrm{SU}(N), \Psi^{\text {new }}$ should be approximately proportional to the $\mathrm{SU}(2)$ wave function in the relative bosonic and fermionic coordinates $\left(x^{i}-y^{i}, \theta_{\alpha}-\eta_{\alpha}\right)$. Motivated by this, we now make a proposal for $\Psi_{0}$ (or equivalently for $\Psi^{\text {new }}$ ) which will be an exact solution of $P_{0} Q_{\alpha}^{1} \Psi_{0}=0$, and satisfies this factorization criterion.

We will in fact define recursively a weighted $n$-body asymptotic wave function,

$$
\Psi_{k_{1}, k_{2}, \cdots, k_{n}}^{(n)}\left(\vec{r}_{1}, \widehat{\theta}_{1 \alpha} ; \vec{r}_{2}, \widehat{\theta}_{2 \alpha} ; \cdots ; \vec{r}_{n}, \widehat{\theta}_{n \alpha}\right)
$$

Here $k_{a}$ are a set of positive integers. By writing $\widehat{\theta}_{\alpha a}$ in the argument, we simply mean that the fermionic component of the wave function is built by quantization of $\widehat{\theta}_{\alpha a}$ according to their appropriate anti-commutators. We will see in the construction below that $\widehat{\theta}_{\alpha a}$ obey the anti-commutation relations

$$
\left\{\widehat{\theta}_{\alpha a}, \widehat{\theta}_{b \beta}\right\}=\frac{1}{k_{a}} \delta_{a b} \delta_{\alpha \beta}
$$

In fact, by construction $\Psi_{k_{1}, \cdots, k_{n}}$ will be a function of the relative positions $\vec{r}_{a}-\vec{r}_{b}$ only, and its fermion component will be built out of $\widehat{\theta}_{\alpha a}-\widehat{\theta}_{\alpha b}$ only.

First of all, we define a two-body wave function,

$$
\Psi_{k_{1}, k_{2}}^{(2)}\left(\vec{r}_{1}, \widehat{\theta}_{\alpha 1} ; \vec{r}_{2}, \widehat{\theta}_{\alpha 2}\right)=C_{k_{1}, k_{2}} \Psi_{\mathrm{SU}(2)}^{\mathrm{new}}\left(\frac{\vec{r}_{1}-\vec{r}_{2}}{\sqrt{k_{1}^{-1}+k_{2}^{-1}}}, \frac{\widehat{\theta}_{\alpha 1}-\widehat{\theta}_{\alpha 2}}{\sqrt{k_{1}^{-1}+k_{2}^{-1}}}\right) .
$$

Here $\Psi_{\mathrm{SU}(2)}^{\text {new }}(\vec{r}, \theta)$ is as in the $\mathrm{SU}(2)$ case,

$$
\Psi_{\mathrm{SU}(2)}^{\text {new }}(\vec{r}, \theta)=\sum_{i, j=1}^{9} \partial_{i} \partial_{j}|\vec{r}|^{-7}\left|s_{i j}\right\rangle_{\theta}
$$

$C_{k_{1}, k_{2}}=C_{k_{2}, k_{1}}$ is a normalization constant that may depend on $k_{1}, k_{2}$, which is so far undetermined. Note that the two-body wave function factor is invariant under exchanging the two bodies $(\vec{r} \rightarrow-\vec{r}, \theta \rightarrow-\theta)$.

Now we define the recursive relation between the $n$-body wave function and the $(n-1)$ body wave function

$$
\begin{aligned}
& \Psi_{k_{1}, k_{2}, \cdots, k_{n}}^{(n)}\left(\vec{r}_{1}, \widehat{\theta}_{1 \alpha} ; \vec{r}_{2}, \widehat{\theta}_{2 \alpha} ; \cdots ; \vec{r}_{n}, \widehat{\theta}_{n \alpha}\right)=\sum_{1 \leq i<j \leq n} C_{k_{i}, k_{j}} \Psi_{\mathrm{SU}(2)}^{\text {new }}\left(\frac{\vec{r}_{i}-\vec{r}_{j}}{\sqrt{k_{i}^{-1}+k_{j}^{-1}}}, \frac{\widehat{\theta}_{i}-\widehat{\theta}_{j}}{\sqrt{k_{i}^{-1}+k_{j}^{-1}}}\right)
\end{aligned}
$$

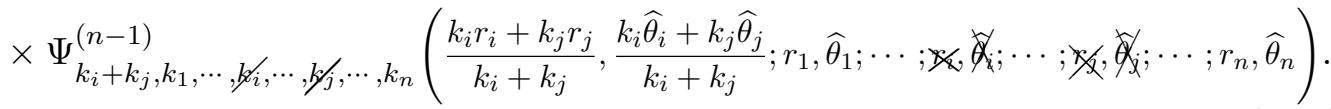


Note that by our construction, $\frac{\widehat{\theta}_{i}-\widehat{\theta}_{j}}{\sqrt{k_{i}^{-1}+k_{j}^{-1}}}$ anti-commutes with $\frac{k_{i} \widehat{\theta}_{i}+k_{j} \widehat{\theta}_{j}}{k_{i}+k_{j}}$ and with all other $\widehat{\theta}_{k}, k \neq i, j$.

It is then straightforward to verify that

$$
\Psi^{\text {new }}=\Psi_{1,1, \cdots, 1}^{(N)}\left(\vec{r}_{1}, \theta_{1 \alpha} ; \vec{r}_{2}, \theta_{2 \alpha} ; \cdots ; \vec{r}_{N}, \theta_{N \alpha}\right)
$$

is an exact solution for the asymptotic ground state Cartan wave function, namely the corresponding $\Psi_{0}^{C}$ is annihilated by $\widetilde{Q}_{\alpha}^{1}{ }^{6}$

The proposed $\Psi^{\text {new }}$ is also manifestly invariant under the permutation (Weyl group action) by $S_{N}$, and is $\mathrm{SO}(9)$ rotationally invariant. And it satisfies the factorization property in various limits of the Coulomb branch with the symmetry breaking pattern $\mathrm{SU}(N) \rightarrow \mathrm{SU}(k) \times \mathrm{SU}(N-k)$. To see the latter, consider the limit where say a cluster $\vec{r}_{1}, \cdots, \vec{r}_{k} \sim \vec{R}_{1}$ are far separated from $\vec{r}_{k+1}, \cdots, \vec{r}_{N} \sim \vec{R}_{2}$. In this limit $\Psi^{\text {new }}$ is dominated by

$$
\begin{gathered}
\Psi^{\text {new }} \longrightarrow C_{k, N-k} \Psi_{\mathrm{SU}(2)}^{\text {new }}\left(\sqrt{\frac{k(N-k)}{N}}\left(\vec{R}_{1}-\vec{R}_{2}\right), \sqrt{\frac{N-k}{k N}}\left(\widehat{\theta}_{1}+\cdots+\widehat{\theta}_{k}\right)\right. \\
\left.-\sqrt{\frac{k}{(N-k) N}}\left(\widehat{\theta}_{k+1}+\cdots+\widehat{\theta}_{N}\right)\right) \\
\times \Psi_{1, \cdots, 1}^{(k)}\left(r_{1}, \widehat{\theta}_{1} ; \cdots ; r_{k}, \widehat{\theta}_{k}\right) \Psi_{1, \cdots, 1}^{(N-k)}\left(r_{k+1}, \widehat{\theta}_{k+1} ; \cdots ; r_{N}, \widehat{\theta}_{N}\right),
\end{gathered}
$$

which scales like $\left|\vec{R}_{1}-\vec{R}_{2}\right|^{-9}$ at large separations between the two clusters. The contributions from other terms in the recursive sum die off like $\left|\vec{R}_{1}-\vec{R}_{2}\right|^{-18}$ or faster in this limit.

$\Psi^{\text {new }}$ may also be expressed as a summation over all trees that join the $N$ particles, the product of two-body wave functions associated with each bifurcation of the tree, weighed by the coefficient $\prod_{\text {bifurcation }} C_{k_{i}, k_{j}}$.

Note that the asymptotic wave function $\Psi_{0}$ is not normalizable, obviously, since it is homogeneous under the simultaneous rescaling of all $\vec{r}_{a}$. We don't have an a priori argument to fix the coefficients $C_{k_{1}, k_{2}}$. It is perhaps tempting to suggest that $C_{k_{1}, k_{2}}=1$ for all $k_{1}, k_{2}$, but this need not be the case. Even though the full two-body wave function has a natural normalization, $\Psi_{k_{1}, k_{2}}^{(2)}$ only captures its tail at large distances.

${ }^{6}$ This is easily seen from the simple identity under the change of variables

$$
\begin{array}{ll}
r^{-}=\frac{r_{1}-r_{2}}{\sqrt{k_{1}^{-1}+k_{2}^{-1}}}, & r^{+}=\frac{k_{1} r_{1}+k_{2} r_{2}}{k_{1}+k_{2}}, \\
\theta^{-}=\frac{\widehat{\theta}_{1}-\widehat{\theta}_{2}}{\sqrt{k_{1}^{-1}+k_{2}^{-1}}}, & \theta^{+}=\frac{k_{1} \widehat{\theta}_{1}+k_{2} \widehat{\theta}_{2}}{k_{1}+k_{2}},
\end{array}
$$

that

$$
\widehat{\theta}_{1} \frac{\partial}{\partial r_{1}}+\widehat{\theta}_{2} \frac{\partial}{\partial r_{2}}=\theta^{-} \frac{\partial}{\partial r^{-}}+\theta^{+} \frac{\partial}{\partial r^{+}} .
$$

The normalization factors are needed in order to preserve the desired normalization of the anti-commutators of $\widehat{\theta}$ 's. 
This proposal would easily answer the question of the overall scaling exponent in $r$. $\Psi^{\text {new }}$ scales like $r^{-9(N-1)}$, and therefore

$$
\kappa=-\frac{3}{2} N(N-1)+9(N-1) .
$$

The power of convergence in the integration of the squared wave function at large $r$ is then $r^{-9(N-1)}$. This is different from the previous proposal of [19] in the $\mathrm{SU}(3)$ case, for instance. The ansatz of [19] is constructed by taking an $\vec{r}_{a}$-independent $\mathrm{SO}(9)$ singlet fermion wave function, multiplied by the scalar harmonic function $r^{-9(N-1)+2}$, and then acted on by all 16 free supercharges $Q_{\alpha}^{\text {new }}$. The resulting wave function falls off faster than our proposal by a factor of $r^{-14}$ at large distances.

\section{Going to higher orders}

\subsection{The general structure}

Now that we have found a solution for $\Psi_{0}$ that obeys

$$
P_{0} Q_{\alpha}^{1} \Psi_{0}=0
$$

we can then determine $\Psi_{1}$ as

$$
\Psi_{1}=\frac{1}{16 H^{0}} Q_{\alpha}^{0} Q_{\alpha}^{1} \Psi_{0}+\mathcal{K}_{1}
$$

where $-16 H^{0}=Q_{\alpha}^{0} Q_{\alpha}^{0}$ (this comes from $\left\{Q_{\alpha}^{0}, Q_{\beta}^{0}\right\}=-2 H^{0} \delta_{\alpha \beta}+\Gamma_{\alpha \beta}^{k} \mathcal{M}_{k}^{0}$ ), and $\mathcal{K}_{1}$ is a yet to be determined wave function in the kernel of $H^{0}$ (or of the $Q_{\alpha}^{0}$ 's). It follows from the Jacobi identity on the $Q_{\alpha}$ 's expanded to first order that (4.2) indeed solves the equation $Q_{\beta}^{0} \Psi_{1}+Q_{\beta}^{1} \Psi_{0}=0$.

The next equation in the $r^{-\frac{3}{2}}$ expansion is

$$
Q_{\alpha}^{0} \Psi_{2}+Q_{\alpha}^{1} \Psi_{1}+Q_{\alpha}^{2} \Psi_{0}=0
$$

Not knowing $\Psi_{2}$, we can again project by $P_{0}$, and consider

$$
P_{0} Q_{\alpha}^{1} \Psi_{1}+P_{0} Q_{\alpha}^{2} \Psi_{0}=0
$$

This may be expressed as an equation for $\mathcal{K}_{1}$,

$$
P_{0} Q_{\alpha}^{1} \mathcal{K}_{1}=-P_{0}\left(Q_{\alpha}^{1} \frac{1}{16 H^{0}} Q_{\beta}^{0} Q_{\beta}^{1}+Q_{\alpha}^{2}\right) \Psi_{0}
$$

The situation here is similar to the equations for $\Psi_{0}$. We could demand $\mathcal{K}_{1}$ to be a Cartan wave function tensored with $\left|\psi_{0}(\widehat{r})\right\rangle$ (the unique ground state of $H^{0}$ in the $(y, \Theta)$ sector), and then try to solve a Dirac-like equation for free superparticles, but now with a source term.

In fact, the r.h.s. of (4.5) vanishes. This can be seen by inspecting the general structure of the r.h.s. of (4.5). $Q_{\beta}^{0} Q_{\beta}^{1} \Psi_{0}$ is a linear combination of states in the $(y, \Theta)$ sector that 
has $H^{0}$ eigenvalues $\frac{1}{2}\left|r_{a b}\right|,\left|r_{a b}\right|$, or $\frac{3}{2}\left|r_{a b}\right|$. It is straightforward to compute $\left(H^{0}\right)^{-1} Q_{\beta}^{0} Q_{\beta}^{1} \Psi_{0}$ explicitly, which we defer to the next subsection. When we act on it further with $P_{0} Q_{\alpha}^{1}$, only the $(y, \Theta)$-sector lowering operators in $Q_{\alpha}^{1}$ contribute. In the end, we can write $P_{0} Q_{\alpha}^{1}\left(H^{0}\right)^{-1} Q_{\beta}^{0} Q_{\beta}^{1} \Psi_{0}$ in a way such that no $\vec{r}_{a}$-derivatives are taken on $\Psi_{0}$. Now $Q_{\alpha}^{0}$ changes the total level in the $y$-sector by an odd amount, while $Q_{\alpha}^{1}$ contains only terms that change the total $y$-level by an even amount. Thus $Q_{\alpha}^{1}\left(H^{0}\right)^{-1} Q_{\beta}^{0} Q_{\beta}^{1} \Psi_{0}$ must be excited in the $y$-sector and is annihilated by $P_{0}$.

As for the term $P_{0} Q_{\alpha}^{2} \Psi_{0}$ on the r.h.s. of (4.5), once again we need only consider the terms in $Q_{\alpha}^{2}$ that leave the $(y, \Theta)$ sector in its ground state. It is not hard to see that $Q_{\alpha}^{2}$ has the schematic form $\theta y \partial_{r}+\theta y^{2} \partial_{y}+\Theta y \partial_{y}+y \Theta^{3}+y \theta \Theta^{2}+y^{3} \partial_{y} \Theta$. The last term comes from expanding $\partial q_{a b}^{j} / \partial X^{i}$ to one order higher than what is computed explicitly in appendix A. We don't need its explicit form nonetheless. None of these terms could keep both $y$ and $\Theta$ sectors in their ground states. We conclude that $P_{0} Q_{\alpha}^{2} \Psi_{0}=0$.

So in the end $\mathcal{K}_{1}$ obeys exactly the same equations as that of $\Psi_{0}$, and can be set to zero. ${ }^{7}$

\subsection{Solving for $\Psi_{1}$}

The next-to-leading order asymptotic wave function $\Psi_{1}$ is thus given by $\frac{1}{16}\left(H^{0}\right)^{-1} Q_{\alpha}^{0} Q_{\alpha}^{1} \Psi_{0}$. We can put $Q_{\alpha}^{1} \Psi_{0}=\left(1-P_{0}\right) Q_{\alpha}^{1} \Psi_{0}$ into the form

$$
\begin{aligned}
Q_{\alpha}^{1} \Psi_{0}=\sum_{a \neq b}\left[\frac{\widehat{r}_{a b}^{j}}{4\left|r_{a b}\right|}\left(\Theta_{a b}^{+} \Gamma^{i j} \Theta_{b a}^{+}\right) \Gamma_{\alpha \beta}^{i} \theta_{\beta a}\right. \\
+\frac{\widehat{r}_{a b}^{i}}{2\left|r_{a b}\right|}\left(y_{a b}^{j} \frac{\partial}{\partial y_{a b}^{j}}+4\right) \Gamma_{\alpha \beta}^{i}\left(\theta_{\beta a}-\theta_{\beta b}\right)+\sum_{c \neq a, b} \frac{y_{a c}^{i} y_{c b}^{j}}{\left|r_{a c}\right|^{\frac{1}{2}}\left|r_{b c}\right|^{\frac{1}{2}}} \Gamma_{\alpha \beta}^{i j}\left(\Theta_{\beta}^{+}\right)_{b a} \\
+\frac{y_{a b}^{i} y_{b a}^{j}}{2\left|r_{a b}\right|} \Gamma_{\alpha \beta}^{i j}\left(\theta_{\beta a}-\theta_{\beta b}\right)-\frac{\widehat{r}_{a b}^{i}}{\left|r_{a b}\right|}\left(1-P_{0}\right) \Gamma_{\alpha \beta}^{i}\left(\Theta_{\beta}\right)_{b a} M_{a b} \\
\left.\quad-\sum_{c \neq a, b}\left(\frac{\left|r_{b c}\right|^{\frac{1}{2}}}{\left|r_{a c}\right|^{\frac{1}{2}}} y_{c a}^{k} \Pi_{c b}^{k j} \frac{\partial}{\partial y_{c b}^{j}}-\frac{\left|r_{a c}\right|^{\frac{1}{2}}}{\left|r_{b c}\right|^{\frac{1}{2}}} y_{b c}^{k} \Pi_{a c}^{k j} \frac{\partial}{\partial y_{a c}^{j}}\right) \frac{\widehat{r}_{a b}^{i}}{\left|r_{a b}\right|^{i}} \Gamma_{\alpha \beta}^{i}\left(\Theta_{\beta}^{+}\right)_{a b}\right] \Psi_{0} .
\end{aligned}
$$

It is straightforward though tedious to compute $Q_{\alpha}^{0} Q_{\alpha}^{1} \Psi_{0}$. By inspecting the excitation levels in the $(y, \Theta)$-sector, we can easily act $\left(H^{0}\right)^{-1}$ on it and obtain, after some simplification,

$$
\begin{gathered}
\Psi_{1}=-\frac{5}{8} \sum_{a \neq b} \frac{1}{\left|r_{a b}\right|^{\frac{3}{2}}}\left(\Theta_{b a}^{+} y_{a b}\left(\theta_{a}-\theta_{b}\right)\right) \Psi_{0}+\sum_{a \neq b} \sum_{c \neq a, b} \frac{1}{\left|r_{a b}\right|+\left|r_{a c}\right|+\left|r_{b c}\right|}\left[\frac{15}{8} \frac{\left(\Theta_{b c}^{+} y_{c a} \Theta_{a b}^{+}\right)}{\left|r_{a c}\right|^{\frac{1}{2}}}\right. \\
\left.+\frac{1}{16}\left(\frac{1}{\left|r_{b c}\right|}-\frac{1}{\left|r_{a b}\right|}\right) \frac{\left(\vec{r}_{a b} \cdot \vec{y}_{c a}\right)\left(\Theta_{a b}^{+} \Theta_{b c}^{+}\right)}{\left|r_{a c}\right|^{\frac{1}{2}}}-2 \frac{\left(\vec{r}_{c b} \cdot \vec{y}_{a c}\right)\left(\vec{y}_{c b} \cdot \vec{y}_{b a}\right)}{\left|r_{a b}\right|^{\frac{1}{2}}\left|r_{a c}\right|^{\frac{1}{2}}\left|r_{b c}\right|^{\frac{1}{2}}}\right] \Psi_{0} .
\end{gathered}
$$

\footnotetext{
${ }^{7}$ More precisely, it can be absorbed into $\Psi_{0}$, which isn't a priori homogeneous. Though our proposal for $\Psi_{0}$ is homogeneous with respect to the simultaneous rescaling of all $\vec{r}_{a}$, in principle there could be corrections of subleading power in $r$, for instance the type of solution considered in [19].
} 


\subsection{Higher orders in the $r^{-\frac{3}{2}}$ expansion}

While the first order correction $\Psi_{1}$ is determined algebraically from $\Psi_{0}$, this is a priori not the case at higher orders. For instance, in order to solve for $\Psi_{2}$, we need to consider the following two equations. The first one is

$$
\begin{aligned}
Q_{\alpha}^{0} \Psi_{2}+ & Q_{\alpha}^{1} \Psi_{1}+Q_{\alpha}^{2} \Psi_{0}=0 \\
& \Rightarrow \Psi_{2}=\frac{1}{16 H^{0}}\left(Q_{\alpha}^{0} Q_{\alpha}^{1} \Psi_{1}+Q_{\alpha}^{0} Q_{\alpha}^{2} \Psi_{0}\right)+\mathcal{K}_{2},
\end{aligned}
$$

where $\mathcal{K}_{2}$ obeys $Q_{\alpha}^{0} \mathcal{K}_{2}=0$. Here we are separating $\Psi_{2}$ into a piece that involves excited states in the off-diagonal $(y, \Theta)$ sector, and a piece $\mathcal{K}_{2}$ that involves only the ground state in the off-diagonal sector. The second equation we need to consider is

$$
\begin{aligned}
& Q_{\alpha}^{0} \Psi_{3}+Q_{\alpha}^{1} \Psi_{2}+Q_{\alpha}^{2} \Psi_{1}+Q_{\alpha}^{3} \Psi_{0}=0 \\
& \quad \Rightarrow P_{0} Q_{\alpha}^{1} \Psi_{2}+P_{0} Q_{\alpha}^{2} \Psi_{1}+P_{0} Q_{\alpha}^{3} \Psi_{0}=0
\end{aligned}
$$

$\mathcal{K}_{2}$ can now be determined from

$$
P_{0} Q_{\alpha}^{1} \mathcal{K}_{2}=-P_{0} Q_{\alpha}^{1} \frac{1}{16 H^{0}}\left(Q_{\beta}^{0} Q_{\beta}^{1} \Psi_{1}+Q_{\beta}^{0} Q_{\beta}^{2} \Psi_{0}\right)-P_{0}\left(Q_{\alpha}^{2} \Psi_{1}+Q_{\alpha}^{3} \Psi_{0}\right) .
$$

The r.h.s. of (4.10) appears to be nontrivial, and now we need to solve a Dirac-like equation for the wave function of $N-1$ superparticles with a source. Note that while we demand $\mathcal{K}_{2}$ to fall off like $r^{-3}$ faster than $\Psi_{0}$ at large distances, $\mathcal{K}_{2}$ is of course not normalizable and such a solution generally exists.

\section{Discussion}

The observation that the leading asymptotic ground state wave function $\Psi_{0}$ is governed by supercharges for free superparticles has been pointed out previously in $[6,20]$. This is perhaps obvious already from the perspective of effective field theory, though in the effective field theory approach it may not have been clear how to construct a systematic asymptotic expansion. In the well known perturbative computation of scattering at large impact parameters [7-10], beyond one-loop order one encounters infrared divergences, which have been mostly ignored. ${ }^{8}$

The condition $P_{0} Q_{\alpha}^{1} \Psi_{0}=0$ does not uniquely determine $\Psi_{0}$, however. If we had started with the wrong ansatz for $\Psi_{0}$, in principle there could be obstructions in solving the recursive equations for the asymptotic expansion at higher orders, or it could also be that the inconsistency is not visible at the level of the asymptotic expansion, but rather may be seen only after summing up the entire series in some way. It would also be tricky to guess a solution that is consistent with all symmetries of the problem. Our proposal is the simplest one that is consistent with all symmetries of the problem and the expected factorization property when the eigenvalues/D0-branes are divided into clusters on the

\footnotetext{
${ }^{8}$ The point is that an IR divergence due to propagators at near zero frequency would have been cut off non-perturbatively, essentially due to the normalizability of the ground state wave function itself.
} 
Coulomb branch. There could be corrections to this proposal already at leading order, namely in $\Psi_{0}$ itself, but it does not seem easy to construct another solution with the desired symmetry properties. [19] suggested a different form of $\Psi_{0}$, which in principle could enter as a correction to our proposal, but it has a different scaling in $r$ and dies off faster at large distances. Even if such corrections are present in $\Psi_{0}$, it would not be possible to determine it based on the asymptotic expansion alone, as it would render $\Psi_{0}$ inhomogeneous under the overall scaling of $r .^{9}$

The structure of the proposed $\Psi_{0}$ may provide some hints on the semi-classical nature of the bulk spacetime, at distances $r \ll N^{\frac{1}{3}}$ (in M-theory Planck units) from the origin. While $N^{\frac{1}{7}} \ll r \ll N^{\frac{1}{3}}$ is the weakly curved type IIA string theory regime, and $1 \ll r \ll N^{\frac{1}{7}}$ is the weakly curved 11-dimensional M-theory regime, both lie in the strong 't Hooft coupling domain of the matrix quantum mechanics, and within the expected spatial spread of the ground state wave function. It has been mysterious why a probe eigenvalue that comes in from the asymptotic region (corresponding to a highly stringy regime in the bulk IIA picture) and interact with the ground state wave function of the remaining, say, $\mathrm{SU}(N-1)$ part of the matrix quantum mechanics, would behave like a semi-classical particle governed by the Born-Infeld action in the bulk geometry. In our proposal for $\Psi_{0}$, which takes the form of a sum over products of two-body wave functions, one could hope the answer to be already approximately valid for $r_{a b}$ 's that are parameterically large compared to 1 (or the scale set by $g_{Y M}$ in the QM), as opposed to $N^{\frac{1}{3}}$ (or $N^{\frac{1}{7}}$ for that matter), though this is not at all obvious. Based on this form of $\Psi_{0}$ and its subleading corrections, perhaps a more reliable computation can be performed for the scattering of eigenvalues/D0-branes off the ground state wave function at impact parameters less than $N^{\frac{1}{3}}$, extending the results of $[8,10]$ to the seemingly non-perturbative regime. ${ }^{10}$

Eventually, we would like to count and understand the structure of long-lived metastable states of the matrix quantum mechanics at large $N$, which are supposed to be dual to microstates of the black hole in the bulk, either in the weakly coupled IIA regime or in the M-theory regime. Despite some numerical success based on Monte Carlo study of the thermal free energy [25-30], there is little analytic understanding of the structure of such nonzero energy states. Some encouraging results are obtained using truncated Schwinger-Dyson equations and extrapolating to the low temperature regime [21-24]. We hope a more precise understanding of the ground state wave function will provide insight on how to construct the general metastable excited states and ultimately a way to study Lorentzian observables relevant to the physics of black holes.

\section{Acknowledgments}

We are grateful to Joe Polchinski for discussions. We would like to thank the organizers of the KITP program New Methods in Nonperturbative Quantum Field Theory, and the

\footnotetext{
${ }^{9}$ Note in particular that $r^{-14}$ is not an integer power of $r^{-\frac{3}{2}}$.

${ }^{10}$ Even in the eikonal regime, taking into account the infrared modification of the propagators due to the ground state wave function already pollutes the structure of an analytic series expansion of the effective potential in $v^{2} / r^{4}$ and in $1 / r^{3}$. This starts at $v^{8}$ order where the $r$ dependence is no longer fixed by supersymmetry.
} 
support of KITP during the course of this work. XY is supported by a Sloan Fellowship and by a Simons Investigator Award from the Simons Foundation. This work is supported in part by the Fundamental Laws Initiative Fund at Harvard University, and by NSF Award PHY-0847457.

\section{A Change of variables in the asymptotic expansion}

Due to the constraint

$$
\vec{q}_{a b} \cdot \vec{r}_{a b}=0
$$

we are only allowed to use

$$
\left(\delta^{i j}-\widehat{r}_{a b}^{i} \widehat{r}_{a b}^{j}\right) \frac{\partial}{\partial q_{a b}^{j}},
$$

where $\widehat{r}_{a b} \equiv \vec{r}_{a b} /\left|\vec{r}_{a b}\right|$.

Writing $X^{i}=U^{-1}\left(r_{a}^{i} E_{a}+q_{a b}^{i} T_{a b}\right) U$, we have

$$
U d X^{i} U^{-1}=\left[r_{a}^{i} E_{a}+q_{a b}^{i} T_{a b}, d U U^{-1}\right]+d r_{a}^{i} E_{a}+d q_{a b}^{i} T_{a b}
$$

Taking the trace of both sides multiplied by $\left(r_{c}^{i}-r_{d}^{i}\right) T_{d c}=r_{c d}^{i} T_{d c}$ (not summing over $c, d$ ), we have

$$
\begin{aligned}
r_{c d}^{i}\left(U d X^{i} U^{-1}\right)_{c d} & =r_{c d}^{i} \operatorname{Tr}\left(T_{d c}\left[r_{a}^{i} E_{a}+q_{a b}^{i} T_{a b}, d U U^{-1}\right]\right) \\
& =\left|r_{c d}\right|^{2}\left(d U U^{-1}\right)_{c d}+r_{c d}^{i}\left[q_{c b}^{i}\left(d U U^{-1}\right)_{b d}-\left(d U U^{-1}\right)_{c b} q_{b d}^{i}\right]
\end{aligned}
$$

The second term on the r.h.s. is down by a factor of $r^{-\frac{3}{2}}$ compared to the first term on the r.h.s., once we make the change of variables $q_{a b}^{i}=\left|r_{a b}\right|^{-\frac{1}{2}} y_{a b}^{i}$ and maintain $y \sim \mathcal{O}(1)$. We can then express

$$
\begin{aligned}
d U U^{-1}= & \sum_{c \neq d} \frac{r_{c d}^{i}}{r_{c d}^{2}}\left(U d X^{i} U^{-1}\right)_{c d} T_{c d} \\
& -\sum_{c \neq d} \frac{r_{c d}^{i}}{r_{c d}^{2}} \sum_{b \neq c, d}\left[\frac{q_{c b}^{i} r_{b d}^{j}}{r_{b d}^{2}}\left(U d X^{j} U^{-1}\right)_{b d}-\frac{q_{b d}^{i} r_{c b}^{j}}{r_{c b}^{2}}\left(U d X^{j} U^{-1}\right)_{c b}\right] T_{c d}+\mathcal{O}\left(r^{-4}\right)
\end{aligned}
$$

Note that the diagonal components of $d U U^{-1}$ are unconstrained and are simply set to zero. Plugging this back into (A.3), we have

$$
\begin{aligned}
U d X^{i} U^{-1}= & \frac{r_{a b}^{i} r_{a b}^{j}}{r_{a b}^{2}}\left(U d X^{i} U^{-1}\right)_{a b} T_{a b}+d r_{a}^{i} E_{a}+d q_{a b}^{i} T_{a b}+\frac{q_{a b}^{i} r_{b a}^{j}}{\left|r_{a b}\right|^{2}}\left(U d X^{j} U^{-1}\right)_{b a}\left(E_{a}-E_{b}\right) \\
& +\sum_{c \neq a, b} \Pi_{a b}^{i j}\left[\frac{q_{a c}^{j} r_{c b}^{k}}{r_{c b}^{2}}\left(U d X^{k} U^{-1}\right)_{c b}-\frac{q_{c b}^{j} r_{a c}^{k}}{r_{a c}^{2}}\left(U d X^{k} U^{-1}\right)_{a c}\right] T_{a b}+\mathcal{O}\left(r^{-3}\right) .
\end{aligned}
$$


From this, we then solve for $d r_{a}^{i}$ and $d q_{a b}^{i}$ in terms of $d X^{i}$ up to $\mathcal{O}\left(r^{-3}\right)$ terms.

$$
\begin{aligned}
d r_{a}^{i}= & \operatorname{Tr}\left(E_{a} U d X^{i} U^{-1}\right)+\sum_{b \neq a} \frac{r_{a b}^{j}}{\left|r_{a b}\right|^{\frac{5}{2}}}\left[y_{a b}^{i}\left(U d X^{j} U^{-1}\right)_{b a}+y_{b a}^{i}\left(U d X^{j} U^{-1}\right)_{a b}\right]+\mathcal{O}\left(r^{-3}\right), \\
d q_{a b}^{i}= & \Pi_{a b}^{i j}\left(U d X^{i} U^{-1}\right)_{a b} \\
& -\sum_{c \neq a, b} \Pi_{a b}^{i j}\left[\frac{y_{a c}^{j}}{\left|r_{a c}\right|^{\frac{1}{2}}} \frac{\widehat{r}_{c b}^{k}}{\left|r_{c b}\right|}\left(U d X^{k} U^{-1}\right)_{c b}-\frac{y_{c b}^{j}}{\left|r_{c b}\right|^{\frac{1}{2}}} \frac{\widehat{r}_{a c}^{k}}{\left|r_{a c}\right|}\left(U d X^{k} U^{-1}\right)_{a c}\right]+\mathcal{O}\left(r^{-3}\right) .
\end{aligned}
$$

Open Access. This article is distributed under the terms of the Creative Commons Attribution License (CC-BY 4.0), which permits any use, distribution and reproduction in any medium, provided the original author(s) and source are credited.

\section{References}

[1] T. Banks, W. Fischler, S.H. Shenker and L. Susskind, $M$ theory as a matrix model: a conjecture, Phys. Rev. D 55 (1997) 5112 [hep-th/9610043] [INSPIRE].

[2] W. Taylor, M(atrix) theory: matrix quantum mechanics as a fundamental theory, Rev. Mod. Phys. 73 (2001) 419 [hep-th/0101126] [INSPIRE].

[3] J.M. Maldacena, The large- $N$ limit of superconformal field theories and supergravity, Int. J. Theor. Phys. 38 (1999) 1113 [hep-th/9711200] [INSPIRE].

[4] V. Balasubramanian, R. Gopakumar and F. Larsen, Gauge theory, geometry and the large- $N$ limit, Nucl. Phys. B 526 (1998) 415 [hep-th/9712077] [INSPIRE].

[5] L. Susskind, Holography in the flat space limit, hep-th/9901079 [INSPIRE].

[6] J. Polchinski, M theory and the light cone, Prog. Theor. Phys. Suppl. 134 (1999) 158 [hep-th/9903165] [INSPIRE].

[7] K. Becker and M. Becker, A two loop test of M(atrix) theory, Nucl. Phys. B 506 (1997) 48 [hep-th/9705091] [INSPIRE].

[8] K. Becker, M. Becker, J. Polchinski and A.A. Tseytlin, Higher order graviton scattering in M(atrix) theory, Phys. Rev. D 56 (1997) 3174 [hep-th/9706072] [InSPIRE].

[9] K. Becker and M. Becker, On graviton scattering amplitudes in M-theory, Phys. Rev. D 57 (1998) 6464 [hep-th/9712238] [INSPIRE].

[10] R. Helling, J. Plefka, M. Serone and A. Waldron, Three graviton scattering in M-theory, Nucl. Phys. B 559 (1999) 184 [hep-th/9905183] [INSPIRE].

[11] P. Yi, Witten index and threshold bound states of D-branes, Nucl. Phys. B 505 (1997) 307 [hep-th/9704098] [INSPIRE].

[12] S. Sethi and M. Stern, D-brane bound states redux, Commun. Math. Phys. 194 (1998) 675 [hep-th/9705046] [INSPIRE].

[13] G.W. Moore, N. Nekrasov and S. Shatashvili, D particle bound states and generalized instantons, Commun. Math. Phys. 209 (2000) 77 [hep-th/9803265] [INSPIRE]. 
[14] A. Konechny, On asymptotic Hamiltonian for $\mathrm{SU}(N)$ matrix theory, JHEP 10 (1998) 018 [hep-th/9805046] [INSPIRE].

[15] M. Porrati and A. Rozenberg, Bound states at threshold in supersymmetric quantum mechanics, Nucl. Phys. B 515 (1998) 184 [hep-th/9708119] [INSPIRE].

[16] S. Sethi and M. Stern, Invariance theorems for supersymmetric Yang-Mills theories, Adv. Theor. Math. Phys. 4 (2000) 487 [hep-th/0001189] [INSPIRE].

[17] J. Plefka and A. Waldron, On the quantum mechanics of M(atrix) theory, Nucl. Phys. B 512 (1998) 460 [hep-th/9710104] [INSPIRE].

[18] J. Fröhlich, G.M. Graf, D. Hasler, J. Hoppe and S.-T. Yau, Asymptotic form of zero energy wave functions in supersymmetric matrix models, Nucl. Phys. B 567 (2000) 231 [hep-th/9904182] [INSPIRE].

[19] J. Hoppe and J. Plefka, The asymptotic ground state of SU(3) matrix theory, hep-th/0002107 [INSPIRE].

$[20]$ D. Hasler and J. Hoppe, Asymptotic factorization of the ground state for $\mathrm{SU}(N)$ invariant supersymmetric matrix models, hep-th/0206043 [INSPIRE].

[21] D.N. Kabat and G. Lifschytz, Approximations for strongly coupled supersymmetric quantum mechanics, Nucl. Phys. B 571 (2000) 419 [hep-th/9910001] [INSPIRE].

[22] D.N. Kabat, G. Lifschytz and D.A. Lowe, Black hole thermodynamics from calculations in strongly coupled gauge theory, Int. J. Mod. Phys. A 16 (2001) 856 [hep-th/0007051] [INSPIRE].

[23] D.N. Kabat, G. Lifschytz and D.A. Lowe, Black hole entropy from nonperturbative gauge theory, Phys. Rev. D 64 (2001) 124015 [hep-th/0105171] [INSPIRE].

[24] Y.-H. Lin, S.-H. Shao, Y. Wang and X. Yin, A low temperature expansion for matrix quantum mechanics, JHEP 05 (2015) 136 [arXiv: 1304.1593] [INSPIRE].

[25] S. Catterall and T. Wiseman, Towards lattice simulation of the gauge theory duals to black holes and hot strings, JHEP 12 (2007) 104 [arXiv:0706.3518] [INSPIRE].

[26] K.N. Anagnostopoulos, M. Hanada, J. Nishimura and S. Takeuchi, Monte Carlo studies of supersymmetric matrix quantum mechanics with sixteen supercharges at finite temperature, Phys. Rev. Lett. 100 (2008) 021601 [arXiv:0707.4454] [INSPIRE].

[27] S. Catterall and T. Wiseman, Black hole thermodynamics from simulations of lattice Yang-Mills theory, Phys. Rev. D 78 (2008) 041502 [arXiv: 0803.4273] [InSPIRE].

[28] M. Hanada, A. Miwa, J. Nishimura and S. Takeuchi, Schwarzschild radius from Monte Carlo calculation of the Wilson loop in supersymmetric matrix quantum mechanics, Phys. Rev. Lett. 102 (2009) 181602 [arXiv:0811.2081] [INSPIRE].

[29] S. Catterall and T. Wiseman, Extracting black hole physics from the lattice, JHEP 04 (2010) 077 [arXiv: 0909.4947] [INSPIRE].

[30] M. Hanada, J. Nishimura, Y. Sekino and T. Yoneya, Monte Carlo studies of matrix theory correlation functions, Phys. Rev. Lett. 104 (2010) 151601 [arXiv:0911.1623] [INSPIRE]. 